\title{
Molecular dynamics simulation of migration fractionation effect of alkylated carbazoles in limestone reservoirs and its application in the Tarim Basin, NW China
}

\author{
QIUYA HAN ${ }^{1}$ MEIJUN LI ${ }^{1, *}$ XIAOQIANG LIU ${ }^{1,2}$ \\ ZICHENG CAO \\ ${ }^{1}$ State Key Laboratory of Petroleum Resources and \\ Prospecting, College of Geosciences, China University of \\ Petroleum, Beijing 102249, China \\ (*correspondence: meijunli@cup.edu.cn) \\ ${ }^{2}$ College of Chemistry and Environmental Engineering, \\ Sichuan University of Science and Engineering, Zigong \\ 643000, China \\ 3 Exploration and Development Research Institute, \\ Northwest Oilfield Branch Corporation, SINOPEC, \\ Urumqi 830011, Xinjiang, China
}

Carbazole (CA) and its methyl and dimethyl substituted homologues are the common nitrogen-containing polycyclic aromatic compounds in crude oils. The ratio of 4-/1methylcarbazole and 2,5-/1,8-dimethylcarbazole have been substantially appicable in sandstone reservoirs to trace oil migration orientation and pathways. In this study, we focus on geo-chromatographic fractionation effect of aforementioned alkylcarbazoles in limestone reservior. As a practical and effective method, the molecular dynamic (MD) simulation have been successful applied in petroleum organic geochemistry, such as the migration fractionation effect of dibenzofurans. Here we calculated the energy of interaction between some alkycarbazoles and calcite surface using MD simulation. The results show that 4-methylcarbazole (4-MCA) and 2,5-dimethylcarbazole (2,5-DMCA) have a slight preference for being adsorbed on calcite surface over 1methylcarbazole (1-MCA) and 1,8-dimethylcarbazole (1,8DMCA), respectively. In addition, we calculated the solubility of these alkylcarbazoles, which indicates the molecules more easily adsorbed on the calcite surface are less soluble in water. It just right suggests that for the oil-wet reservior the ratio of 4-/1-MCA and 2,5-/1,8-DMCA decrease with increasing migration distance. It is further shown in theory that migration fractionation mechanism of some alkylated carbazole isomers in limestone reservior at the molecular level. These two indicators were also applied successfully in Ordovician carbonate reservoir in the Tuoputai region, Tarim Basin, NW China and can be used as effective molecular markers for tracing migration orientation and pathways. 